\title{
Spontaneous emission can locally create quantum discord out of classical correlations
}

\author{
Marek Gwóźdź • Lech Jakóbczyk
}

Received: 12 July 2013 / Accepted: 5 September 2013 / Published online: 27 September 2013

(C) The Author(s) 2013. This article is published with open access at Springerlink.com

\begin{abstract}
We consider local time evolution given by spontaneous emission in the system of independent two-level atoms. It is shown that all classically correlated initial states are driven into the states with transient non-zero quantum discord. Thus local creation of genuine quantum correlations can be observed in a simple physical system of non-interacting atoms which are not completely isolated from the environment.
\end{abstract}

Keywords Quantum discord · Spontaneous emission · Local generation of quantum discord

\section{Introduction}

Quantum discord [1] is the most promising measure of bipartite quantum correlations beyond celebrated quantum entanglement. Some mixed separable states behave "non-classically" with respect to the local measurements and exactly such states have non zero quantum discord. As it was shown recently, almost all quantum states have non-vanishing discord [2], so an arbitrary small disturbance can drive a classically correlated state into a state with genuine quantum correlations. In contrast to the entanglement, even local noise can increase or create quantum discord $[3,4]$ and it was shown that a trace-preserving local channel can create quantum correlations in some classical states if and only if it is not commutativity preserving channel (see e.g. $[5,6])$.

Although this phenomenon was described in some recent publications (see e.g. [710]) we reconsider it again in a simple and natural physical situation. It turns out that to observe such local creation of quantum correlations it is enough to take two

M. Gwóźdź · L. Jakóbczyk ( $₫)$

Institute of Theoretical Physics, University of Wrocław, Plac Maxa Borna 9,

50-204 Wrocław, Poland

e-mail: lech.jakobczyk@ift.uni.wroc.pl; ljak@ift.uni.wroc.pl 
non-interacting two-level atoms not completely isolated from the environment. Time evolution of the system is given by a dissipative process of spontaneous emission. In the Markov approximation, this time evolution is described by the semi-group of local completely positive mappings i.e. local quantum channels. One can check that the channels are not commutativity preserving, so local creation of quantum correlations can appear. We show that all classically correlated initial states (so called classicalclassical and classical-quantum states) are driven by the process of spontaneous emission into the states with transient non-zero quantum discord. The created discord is transient because during time evolution all correlations asymptotically decay. Apart from the usual spontaneous emission of two atoms, we also consider "one-sided" spontaneous emission in which only one atom emits photons and the other is completely decoupled from the environment. It turns out that in such system, local creation of quantum discord is more effective.

To quantify non-classical correlations in bipartite states which may differ from entanglement, we use geometric quantum discord [11] (see also [12] for other geometric measures of correlations). Classically correlated quantum states have zero discord and we take them as the initial states of the studied evolution. We found it useful to consider also some measure of total correlation present in the system. In our study we propose to use an algebraic measure given by so called maximal mutual correlation $C_{M}$, introduced in the context of the algebraic approach to quantum non-separability [13]. This quantity is closely related to correlation distance of the state [14] i.e. the trace norm distance between given state and the tensor product of its partial traces. It is easy to compute and contains some information about correlations beyond quantum discord. In particular, in the case of classically correlated states $C_{M}>0$ and it encodes the properties of classical probability distributions defining such states (Sect. 2). In our analysis $C_{M}$ plays only an additional role. It can help to choose appropriate initial classical state, since as we show, during the time evolution caused by local noise, part of the initial classical correlations are transformed into genuine quantum correlations and such creation of quantum discord is effective when the initial value of $C_{M}$ is large.

The main purpose of our work is to study time evolution of a measure of quantum correlations. Geometric measure of quantum discord is defined in terms of minimal distance of the given state from the set of classical states, so the proper choice of such a distance is very important. The measure proposed in [11] uses a Hilbert-Schmidt distance in a set of states. In this case the minimization process is simple and can be realized analytically for arbitrary two-qubit states. Despite of this feature, geometric discord based on the Hilbert-Schmidt distance has some unwanted properties. The most serious problem with such a measure is that it may increase under local operations on the unmeasured subsystem $[15,16]$. Fortunately, this defect can be repaired by using other norm in the set of states. As was shown in Ref. [17], the best choice is to use the trace norm (or Schatten 1-norm) in place of Hilbert-Schmidt norm to define geometric discord. Such defined measure will be able to consistently quantify nonclassical correlations, but it is more difficult to compute. The closed formula for it is known only in the case of Bell-diagonal or X-type two-qubit states $[17,18]$. For technical reasons in this paper we adopt the standard definition of geometric discord based on the Hilbert-Schmidt norm. We consider the time evolution of the classical-classical and classical-quantum initial states with the largest possible value 
of maximal mutual correlation $C_{M}$ and we shown that for such states the geometric discord grows from zero to some maximal value and then asymptotically decays. There is no danger that such production of quantum correlations is an artificial effect of sensitivity of the quantum discord to the choice of distance measure, because the evolution always concerns the measured subsystem. Moreover, in the case when the unmeasured subsystem also evolves, the production of correlations is smaller (Sect. 3). The comparison with the local evolution of trace distance discord even more supports the conclusion that this effect is real. Preliminary results of the analysis of this problem show that the trace distance discord is also locally created [19].

\section{Geometric measure of quantum discord and maximal mutual correlation}

We start with the introduction of the standard notion of geometric quantum discord [11]. When a $d \otimes d$ bipartite system $A B$ is prepared in a state $\rho$ and we perform local measurement on the subsystem $A$, almost all states $\rho$ will be disturbed due to such measurement. The (one-sided) geometric discord $D_{G}^{A}(\rho)$ can be defined as the minimal disturbance, measured by the squared Hilbert-Schmidt distance, induced by any projective measurement $\mathbb{P}^{A}$ on subsystem $A$ i.e.

$$
D_{G}^{A}(\rho)=\frac{d}{d-1} \min _{\mathbb{P}^{A}}\left\|\rho-\mathbb{P}^{A}(\rho)\right\|_{2}^{2},
$$

where

$$
\|m\|_{2}=\sqrt{\operatorname{tr}\left(m m^{\dagger}\right)}
$$

Here we adopt normalized version of the geometric discord, introduced in Ref. [20]. There is a subclass of states which are left unperturbed by at least one measurement. Such "classical-quantum" states $\rho_{c q}$ satisfy

$$
\mathbb{P}^{A}\left(\rho_{c q}\right)=\sum_{k}\left(P_{k} \otimes \mathbb{1}\right) \rho_{c q}\left(P_{k} \otimes \mathbb{1}\right)=\rho_{c q}
$$

for some von Neumann measurement $\left\{P_{k}\right\}$. Obviously $D_{G}^{A}\left(\rho_{c q}\right)=0$ and

$$
\rho_{c q}=\sum_{k} p_{k} P_{k} \otimes \rho_{k}^{B},
$$

where $P_{k}=\left|\psi_{k}\right\rangle\left\langle\psi_{k}\right|$ for some orthonormal basis $\left|\psi_{k}\right\rangle, \rho_{k}^{B}$ are quantum states in $B$ and $\left\{p_{k}\right\}$ is the classical probability distribution. The geometric discord can be also interpreted as the distance between the state $\rho$ and the closest classical-quantum state. One can also consider fully classically correlated quantum states (known as "classical-classical" states), defined as

$$
\rho_{c c}=\sum_{j, k} p_{j k} P_{j}^{A} \otimes P_{k}^{B},
$$


where $\left\{P_{j}^{A}\right\}$ and $\left\{P_{k}^{B}\right\}$ are von Neumann measurements on subsystems $A$ and $B$ respectively, and $\left\{p_{j k}\right\}$ is a two-dimensional probability distribution. The states (2.5) satisfy $D_{G}^{A}\left(\rho_{c c}\right)=0$ but also have zero discord over two-sided projective measurements [22].

In the case of two qubits studied in the present paper, there is an explicit expression for $D_{G}^{A}[11]$

$$
D_{G}^{A}(\rho)=\frac{1}{2}\left(\|x\|^{2}+\|T\|_{2}^{2}-k_{\max }\right),
$$

where the components of the vector $\boldsymbol{x} \in \mathbb{R}^{3}$ are given by

$$
x_{k}=\operatorname{tr}\left(\rho \sigma_{k} \otimes \mathbb{1}\right) .
$$

The matrix $T$ has elements

$$
T_{j k}=\operatorname{tr}\left(\rho \sigma_{j} \otimes \sigma_{k}\right)
$$

and $k_{\max }$ is the largest eigenvalue of the matrix $\boldsymbol{x} \boldsymbol{x}^{T}+T T^{T}$. One also finds a simple relation between geometric quantum discord and entanglement measured by normalized negativity

$$
N(\rho)=\left\|\rho^{P T}\right\|_{1}-1,
$$

where $P T$ stands for partial transposition and $\|m\|_{1}=\operatorname{tr}|m|$. The relation is as follows [20]: for all pure states

$$
\sqrt{D_{G}^{A}(|\psi\rangle)}=N(|\psi\rangle)
$$

whereas for every general two-qubit state $\rho$

$$
\sqrt{D_{G}^{A}(\rho)} \geq N(\rho)
$$

Notice that the relation (2.11) is not universal-there are conterexamples in higher dimensional systems [21].

We also shortly discuss the notion of trace distance geometric discord $D_{1}^{A}(\rho)$. This quantity is defined as follows [17]

$$
D_{1}^{A}(\rho)=\min _{\mathbb{P} A}\left\|\rho-\mathbb{P}^{A}(\rho)\right\|_{1} .
$$

In contrast to the geometric discord based on the Hilbert-Schmidt norm, $D_{1}^{A}$ is nonincreasing under general local operations on subsystem $B$, but its computation is much more involved [18]. Also the relation between $D_{G}^{A}$ and $D_{1}^{A}$ is not clear. It can show [17] that for Bell-diagonal states

$$
D_{1}^{A}(\rho) \geq \sqrt{D_{G}^{A}(\rho)},
$$


but for general two-qubit states the inequality (2.13) cannot be true. In the present paper we study time evolution of $D_{G}^{A}$, analogous problem for $D_{1}^{A}$ will be considered elsewhere [19].

In a study of time evolution of classical and quantum correlations in a bipartite systems it is useful to consider some measure of total correlations present in the system. In this paper we propose to use so called maximal mutual correlation $C_{M}(\rho)$, a quantity motivated by the algebraic approach to quantum entanglement [13]. In the algebraic formulation, a bipartite quantum system is defined by the algebra of observables $\mathcal{A}_{\text {tot }}$ with distinguished mutually commuting subalgebras $\mathcal{A}$ and $\mathcal{B}$ corresponding to subsystems. Any state $\rho$ defines a linear functional $\omega_{\rho}$ on the algebra $\mathcal{A}_{\text {tot }}$

$$
\omega_{\rho}(a)=\operatorname{tr}(\rho a), \quad a \in \mathcal{A}_{\text {tot }}
$$

Maximal mutual correlation between the subalgebras $\mathcal{A}$ and $\mathcal{B}$ in the state $\rho$ is defined as

$$
C_{M}(\rho)=\sup _{a, b}\left|\omega_{\rho}(a b)-\omega_{\rho}(a) \omega_{\rho}(b)\right|
$$

In the formula (2.15), the supremum is taken over all elements $a \in \mathcal{A}$ and $b \in \mathcal{B}$ such that $\|a\|=\|b\|=1$. The quantity $C_{M}(\rho)$ indicates how much given state differs from a product state when we take into account only local observables. Notice that

$$
C_{M}(\rho) \leq\left\|\omega_{\rho}-\omega_{\rho}^{A} \otimes \omega_{\rho}^{B}\right\|=\left\|\rho-\rho^{A} \otimes \rho^{B}\right\|_{1}
$$

where the marginal states $\omega_{\rho}^{A}$ and $\omega_{\rho}^{B}$ are given by partial traces $\rho^{A}$ and $\rho^{B}$ of a density matrix $\rho$. Thus maximal mutual correlation is always dominated by the correlation distance $C(\rho)$ given by

$$
C(\rho)=\left\|\rho-\rho^{A} \otimes \rho^{B}\right\|_{1}
$$

This quantity was recently introduced by Hall [14] in the context of analysis of quantum mutual information.

For two qubits we may choose

$$
a=\boldsymbol{a} \cdot \boldsymbol{\sigma} \otimes \mathbb{1}, \quad b=\mathbb{1} \otimes \boldsymbol{b} \cdot \boldsymbol{\sigma},
$$

where $\boldsymbol{a}$ and $\boldsymbol{b}$ are normalized vectors in $\mathbb{R}^{3}$. Then

$$
\omega_{\rho}(a b)-\omega_{\rho}(a) \omega_{\rho}(b)=\langle\boldsymbol{a}, Q \boldsymbol{b}\rangle,
$$

with the correlation matrix $Q=\left(q_{i j}\right)$ defined by

$$
q_{i j}=\operatorname{tr}\left(\sigma_{i} \otimes \sigma_{j} \rho\right)-\operatorname{tr}\left(\sigma_{i} \otimes \mathbb{1} \rho\right) \operatorname{tr}\left(\mathbb{1} \otimes \sigma_{j} \rho\right) .
$$


Thus

$$
C_{M}(\rho)=\sup _{\boldsymbol{a}, \boldsymbol{b}}|\langle\boldsymbol{a}, Q \boldsymbol{b}\rangle|=\|Q\|
$$

and $C_{M}(\rho)$ is given by the matrix norm of the corresponding correlation matrix i.e. the largest singular value of $Q$. It turns out that for any pure state $|\psi\rangle, C_{M}(|\psi\rangle)$ gives the same information as any other measure of quantum correlations. In particular [13]

$$
C_{M}(|\psi\rangle)=\sqrt{D_{G}^{A}(|\psi\rangle)}=N(|\psi\rangle)
$$

For mixed states we expect that

$$
C_{M}(\rho) \geq \sqrt{D_{G}^{A}(\rho)} \geq N(\rho)
$$

The second inequality was shown in Ref. [20] whereas the first one can be proved analytically for Bell-diagonal states and numerical simulations suggest that it is valid for all mixed two-qubit states [23]. The first inequality also indicates that $C_{M}$ contains some information about correlations beyond quantum discord i.e. classical correlations present in the quantum states. For fully classically correlated states $\rho_{c c}$ given by (2.5) one can show that [23]

$$
\begin{aligned}
C_{M}\left(\rho_{c c}\right)= & \mid\left(p_{11}-p_{22}\right)^{2}-\left(p_{12}-p_{21}\right)^{2} \\
& +p_{12}+p_{21}-p_{11}-p_{22} \mid
\end{aligned}
$$

The right hand side of the Eq. (2.24) can be interpreted as the modulus of covariance of two discrete random variables $X$ and $Y$ with values in the set $\{-1,+1\}$ and with join probability distribution defined by probabilities $p_{j k}$ as follows

$$
\begin{aligned}
& \operatorname{Prob}\{X=+1, Y=+1\}=p_{11}, \\
& \operatorname{Prob}\{X=+1, Y=-1\}=p_{12}, \\
& \operatorname{Prob}\{X=-1, Y=+1\}=p_{21}, \\
& \operatorname{Prob}\{X=-1, Y=-1\}=p_{22} .
\end{aligned}
$$

So indeed $\rho_{c c}$ is only classically correlated and

$$
C_{M}\left(\rho_{c c}\right)=|\operatorname{Cov}(X, Y)| \text {. }
$$

On the other hand, for classical-quantum states $\rho_{c q}$ given by (2.4), we obtain

$$
C_{M}\left(\rho_{c q}\right)=2 p_{1} p_{2}\left\|\boldsymbol{a}_{1}-\boldsymbol{a}_{2}\right\|
$$

where $\boldsymbol{a}_{1,2}$ are the Bloch vectors corresponding to the states $\rho_{1,2}^{B}$ i.e.

$$
\rho_{1,2}^{B}=\frac{1}{2}\left(\mathbb{1}+\boldsymbol{a}_{1,2} \cdot \boldsymbol{\sigma}\right) .
$$


The factor $2 p_{1} p_{2}$ in Eq. (2.27) can be connected with statistical properties of discrete random variable $X$ with values in the set $\{-1,+1\}$ and probability distribution

$$
\operatorname{Prob}\{X=+1\}=p_{1}, \quad \operatorname{Prob}\{X=-1\}=p_{2} .
$$

One checks that

$$
2 p_{1} p_{2}=\frac{1}{2}(\operatorname{Var} X)^{2}
$$

so

$$
C_{M}\left(\rho_{c q}\right)=\frac{1}{2}(\operatorname{Var} X)^{2}\left\|\boldsymbol{a}_{1}-\boldsymbol{a}_{2}\right\|
$$

In that case maximal mutual correlation depends not only on the classical probability distribution, but also on the properties of quantum states of the subsystem $B$.

In the next section we will study time evolution of initial states $\rho_{c q}$ and $\rho_{c c}$ in the system of two-level atoms. We consider only spontaneous emission of independent atoms and show that this simple and natural physical process leads to the transient creation of quantum correlations out of classical correlations.

\section{Spontaneous emission in a system of two-level atoms and local generation of quantum discord}

Consider a system of two independent two-level atoms (atom $A$ and atom $B$ ) interacting with environment at zero temperature. When we take into account only the dissipative process of spontaneous emission, the dynamics of the system is given by the master equation [24]

$$
\frac{d \rho}{d t}=L_{A B} \rho, \quad L_{A B}=L_{A}+L_{B},
$$

where for $k=A, B$

$$
L_{k}=\frac{\gamma_{0}}{2}\left(2 \sigma_{-}^{k} \rho \sigma_{+}^{k}-\sigma_{+}^{k} \sigma_{-}^{k} \rho-\rho \sigma_{+}^{k} \sigma_{-}^{k}\right) .
$$

In the above equation $\sigma_{ \pm}^{A}=\sigma_{ \pm} \otimes \mathbb{1}, \sigma_{ \pm}^{B}=\mathbb{1} \otimes \sigma_{ \pm}$and $\gamma_{0}$ is the single atom spontaneous emission rate. Time evolution of the initial density matrix $\rho$ of the system is given by the semi-group $\left\{T_{t}^{A B}\right\}_{t \geq 0}$ generated by $L_{A B}$ and such evolution has a well known properties. Let us remind here the matrix elements of the state $\rho(t)$ with respect to the basis $|e\rangle_{A} \otimes|e\rangle_{B},|e\rangle_{A} \otimes|g\rangle_{B},|g\rangle_{A} \otimes|e\rangle_{B},|g\rangle_{A} \otimes|g\rangle_{B}$ (where $|g\rangle_{k},|e\rangle_{k}, k=$ $A, B$ are the ground states and excited states of atoms $A$ and $B$ )

$$
\begin{aligned}
& \rho_{11}(t)=e^{-2 \gamma_{0} t} \rho_{11}, \\
& \rho_{1 k}(t)=e^{-\frac{3 \gamma_{0}}{2} t} \rho_{1 k}, k=2,3, \\
& \rho_{14}(t)=e^{-\gamma_{0} t} \rho_{14},
\end{aligned}
$$




$$
\begin{aligned}
& \rho_{k k}(t)=\left(e^{-\gamma_{0} t}-e^{-2 \gamma_{0} t}\right) \rho_{11}+e^{-\gamma_{0} t} \rho_{k k}, k=2,3, \\
& \rho_{23}(t)=\left(e^{-\frac{\gamma_{0}}{2} t}-e^{-\frac{3 \gamma_{0}}{2} t}\right) \rho_{13}+e^{-\frac{\gamma_{0}}{2} t} \rho_{24}, \\
& \rho_{34}(t)=\left(e^{-\frac{\gamma_{0}}{2} t}-e^{-\frac{3 \gamma_{0}}{2} t}\right) \rho_{12}+e^{-\frac{\gamma_{0}}{2} t} \rho_{34}
\end{aligned}
$$

and

$$
\rho_{44}(t)=1-\rho_{11}(t)-\rho_{22}(t)-\rho_{33}(t)
$$

The remaining matrix elements can be obtained by the hermiticity condition. The evolution given by the semi-group $\left\{T_{t}^{A B}\right\}_{t \geq 0}$ is ergodic: for any initial state, the asymptotic state is equal to the ground state of two atoms.

To investigate the process of local generation of quantum discord, it is instructive to consider "one-sided" spontaneous emission i.e. time evolution $\left\{T_{t}^{A}\right\}_{t \geq 0}$ generated only by $L_{A}$. Here the atom $A$ spontaneously emits photons, whereas the atom $B$ is isolated from the environment. Such dynamics has peculiar properties, so we discuss it in some details. In that case the state $\rho(t)$ at time $t$ has the following matrix elements

$$
\begin{array}{ll}
\rho_{11}(t)=e^{-\gamma_{0} t} \rho_{11}, & \rho_{12}(t)=e^{-\gamma_{0} t} \rho_{12}, \\
\rho_{22}(t)=e^{-\gamma_{0} t} \rho_{22}, & \rho_{13}(t)=e^{-\frac{\gamma_{0}}{2} t} \rho_{13}, \\
\rho_{14}(t)=e^{-\frac{\gamma_{0}}{2} t} \rho_{14}, & \rho_{23}(t)=e^{-\frac{\gamma_{0}}{2} t} \rho_{23}, \\
\rho_{24}(t)=e^{-\frac{\gamma_{0}}{2} t} \rho_{24} &
\end{array}
$$

and

$$
\begin{aligned}
& \rho_{33}(t)=\left(1-e^{-\gamma_{0} t}\right) \rho_{11}+\rho_{33}, \\
& \rho_{34}(t)=\left(1-e^{\gamma_{0} t}\right) \rho_{12}+\rho_{34}, \\
& \rho_{44}(t)=\left(1-e^{-\gamma_{0} t}\right) \rho_{22}+\rho_{44} .
\end{aligned}
$$

Again the remaining matrix elements can be obtained by the hermiticity condition.

From the Eqs. (3.5) and (3.6) it follows that in contrast to the usual process of spontaneous emission, now there are non-trivial asymptotic states of the system, given by

$$
\rho_{\mathrm{as}}=P_{g} \otimes \operatorname{tr}_{A} \rho
$$

where $P_{g}$ is the projection on the ground state of the atom $A$ and $\rho$ is the initial density matrix.

Notice that in both cases, asymptotic state is a product state, so any measure of quantum correlations should asymptotically vanish during such time evolutions. But the transient quantum correlation of some states still can exist. To show this in an explicit way, consider the initial states $\rho_{c q}$ and $\rho_{c c}$. In the case of two qubits, these states are defined in terms of orthogonalprojectors 


$$
\begin{aligned}
P_{1} & =\left(\begin{array}{ll}
\cos ^{2} \vartheta & \frac{1}{2} e^{-i \varphi} \sin 2 \vartheta \\
\frac{1}{2} e^{i \varphi} \sin 2 \vartheta & \sin ^{2} \vartheta
\end{array}\right) \\
P_{2} & =\left(\begin{array}{ll}
\sin ^{2} \vartheta & -\frac{1}{2} e^{-i \varphi} \sin 2 \vartheta \\
-\frac{1}{2} e^{i \varphi} \sin 2 \vartheta & \cos ^{2} \vartheta
\end{array}\right) .
\end{aligned}
$$

In particular

$$
\rho_{c q}=p_{1} P_{1} \otimes \rho_{1}^{B}+p_{2} P_{2} \otimes \rho_{2}^{B}
$$

where the states $\rho_{1,2}^{B}$ are given by (2.28). Similarly, up to the local unitary equivalence, the states $\rho_{c c}$ can be written as

$$
\rho_{c c}=\sum_{j, k=1}^{2} p_{j k} P_{j} \otimes P_{k} .
$$

Since local dissipative evolution can only degrade maximal mutual correlations and the relation (2.23) is valid for all times, the initial state should have a large value of $C_{M}$. In the case of classically correlated states (3.10), the largest value of $C_{M}\left(\rho_{c c}\right)$ is obtained when for example

$$
p_{11}=p_{22}=0 \text { and } p_{12}=p_{21}=\frac{1}{2}
$$

Detailed analysis of time evolution of such initial states shows that the maximal creation of quantum correlations happens when in the parametrization of orthogonal projections (3.8) we take $\vartheta=\pi / 4$. So we first consider time evolution of the initial state

$$
\rho_{0}=\frac{1}{2}|+\rangle\langle+|\otimes|-\rangle\left\langle-\left|+\frac{1}{2}\right|-\right\rangle\langle-|\otimes|+\rangle\langle+|,
$$

where

$$
| \pm\rangle=\frac{1}{\sqrt{2}}(|e\rangle \pm|g\rangle) .
$$

We study evolution of (3.11) given by the semi-group $\left\{T_{t}^{A B}\right\}_{t \geq 0}$ as well as one-sided evolution defined by $\left\{T_{t}^{A}\right\}_{t \geq 0}$ i.e.

$$
\rho_{0}(t)=T_{t}^{A B} \rho_{0}, \quad \widetilde{\rho}_{0}(t)=T_{t}^{A} \rho_{0} .
$$

In both cases, the maximal mutual correlation decays

$$
C_{M}\left(\rho_{0}(t)\right)=e^{-\gamma_{0} t}, \quad C_{M}\left(\tilde{\rho}_{0}(t)\right)=e^{-\gamma_{0} t / 2},
$$

but this decay is slower for one-sided spontaneous emission. On the other hand

$$
D_{G}^{A}\left(\rho_{0}(t)\right)=\min \left(D_{1}(t), D_{2}(t)\right)
$$


with

$$
\begin{aligned}
& D_{1}(t)=\frac{1}{2} e^{-2 \gamma_{0} t}, \\
& D_{2}(t)=\frac{1}{2}\left[\left(e^{-\gamma_{0} t}-1\right)^{2}+e^{-4 \gamma_{0} t}\left(e^{\gamma_{0} t}-1\right)^{4}\right]
\end{aligned}
$$

and

$$
D_{G}^{A}\left(\widetilde{\rho}_{0}(t)\right)=\min \left(\widetilde{D}_{1}(t), \widetilde{D}_{2}(t)\right)
$$

where

$$
\widetilde{D}_{1}(t)=\frac{1}{2} e^{-\gamma_{0} t}, \quad \widetilde{D}_{2}(t)=\frac{1}{2}\left(e^{-\gamma_{0} t}-1\right)^{2}
$$

As we see, one-sided geometric discord behaves differently then $C_{M}$ during time evolution. In both cases $D_{G}^{A}$ grows from zero to some maximal value and then it asymptotically goes to zero. Moreover, for all $t$

$$
C_{M}\left(\rho_{0}(t)\right) \geq \sqrt{D_{G}^{A}\left(\rho_{0}(t)\right)}
$$

and similarly for one-sided spontaneous emission. Notice also that one-sided spontaneous emission produces more quantum correlations then the usual process of local spontaneous emission of two atoms (Fig. 1). It can be connected with the fact that the time evolution operator is applied only to the part of the system which is measured. On the other hand, one-sided evolution operating only on subsystem $B$ cannot produce non-zero (one-sided) quantum discord $D_{G}^{A}$, since initial classicalclassical or classical-quantum states become classical-quantum states under the evolution. For other initial states of this type, the analysis of time evolution of geometric discord is much more involved. Here we consider only the initial states $\rho_{1}$ and $\rho_{2}$ defined by $\vartheta=\pi / 6$ and $\vartheta=\pi / 8$, respectively. Figure 2 shows evolution of $D_{G}^{A}$ for such initial states, compared to the analogous evolution for the initial state

Fig. 1 Time evolution of the geometric discord of the initial state $\rho_{0}$ due to one-sided spontaneous emission (solid line) and usual spontaneous emission of two atoms (dotted line)

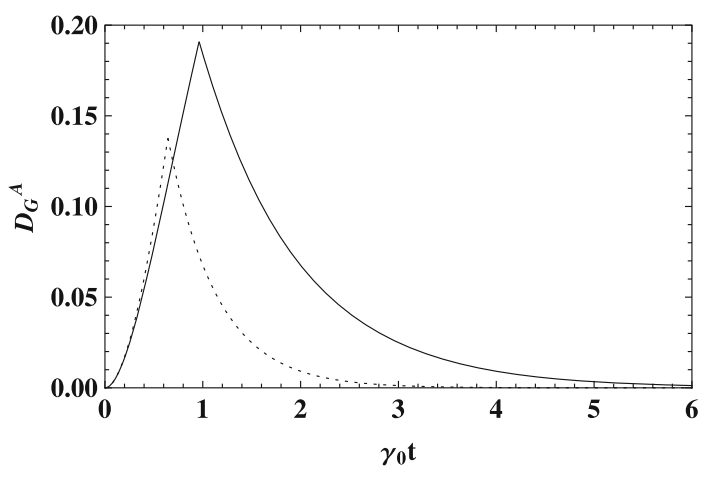


Fig. 2 Evolution of $D_{G}^{A}$ for initial states $\rho_{0}$ (solid line), $\rho_{1}$ (dotted line) and $\rho_{2}$ (dashed line)

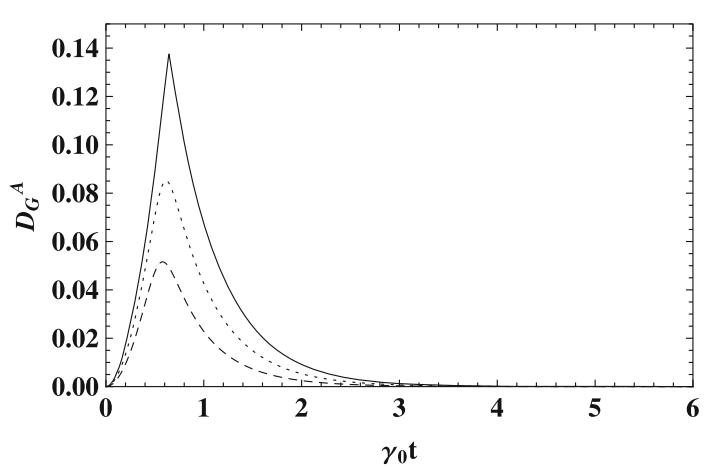

$\rho_{0}$. We see that for such initial states, the creation of quantum discord is less effective.

Consider now $\rho_{c q}$ as the initial state. It follows from the formula (2.31) that for such state the largest value of $C_{M}$ is obtained when $p_{1}=p_{2}=1 / 2$ and the Bloch vectors $\boldsymbol{a}_{1}$ and $\boldsymbol{a}_{2}$ are opposite and normalized i.e. the states $\rho_{1}^{B}$ and $\rho_{2}^{B}$ are orthogonal projectors. Such $\rho_{c q}$ is the specific example of classical-classical state. For the typical classical-quantum state, $C_{M}$ is always less then 1. Direct calculations also show that similarly as in the classical-classical case, the creation of quantum correlations is maximal when the projectors in the "classical" part of $\rho_{c q}$ are chosen to be

$$
P_{1}=|+\rangle\left\langle+\left|, \quad P_{2}=\right|-\right\rangle\langle-|
$$

and the initial state is the following

$$
\rho=\frac{1}{2}|+\rangle\left\langle+\left|\otimes \rho_{1}^{B}+\frac{1}{2}\right|-\right\rangle\langle-| \otimes \rho_{2}^{B} .
$$

The creation of quantum discord still depends on the quantum part of the state $\rho$. To study this dependence, consider the states of the subsystem $B$ defined by normalized Bloch vectors

$$
\boldsymbol{a}_{1}=\left(\cos \alpha_{0}, \sin \alpha_{0}, 0\right), \quad \boldsymbol{a}_{2}=\left(\cos \left(\alpha_{0}+\alpha\right), \sin \left(\alpha_{0}+\alpha\right), 0\right)
$$

Notice that

$$
\left\|\boldsymbol{a}_{1}-\boldsymbol{a}_{2}\right\|^{2}=2(1-\cos \alpha)
$$

where the parameter $\alpha_{0}$ is fixed. For such initial states and dynamics given by spontaneous emission of two atoms, one obtains

$$
D_{G}^{A}(\rho(t))=\min \left(D_{1}^{q}(t), D_{2}^{q}(t)\right)
$$


Fig. 3 Maximal value of created discord versus the angle between Bloch vectors

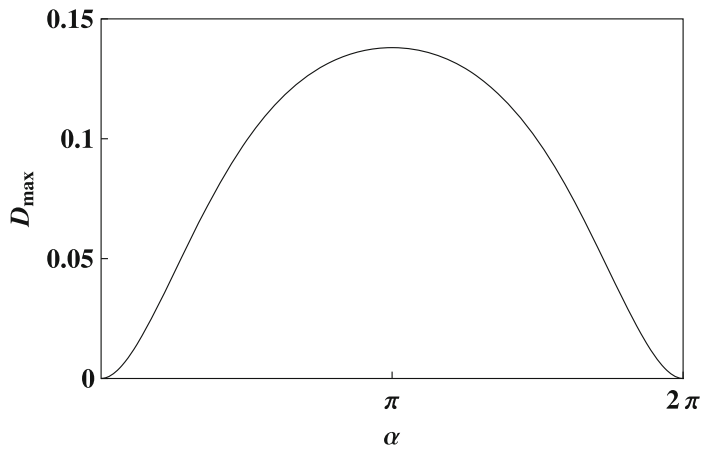

with

$$
\begin{aligned}
D_{1}^{q}(t)= & \frac{1}{4}(1-\cos \alpha) e^{-2 \gamma_{0} t} \\
D_{2}^{q}(t)= & 1+\frac{1}{2} e^{-4 \gamma_{0} t}-\frac{7}{4} e^{-3 \gamma_{0} t}+3 e^{-2 \gamma_{0} t}-\frac{11}{4} e^{-\gamma_{0} t} \\
& +\left(\frac{1}{4} e^{-3 \gamma_{0} t}-\frac{1}{2} e^{-2 \gamma_{0} t}+\frac{1}{4} e^{-\gamma_{0} t}\right) \cos \alpha
\end{aligned}
$$

Again $D_{G}^{A}(\rho(t))$ grows from zero to the maximal value $D_{\max }$ and then decays to zero. The maximum of the created discord depends only on the angle $\alpha$ between the Bloch vectors $\boldsymbol{a}_{1}$ and $\boldsymbol{a}_{2}$ (or a distance between them) i.e. in contrast to the classical part of $\rho$ it does not depend on detailed structure of the states on the subsystem $B$ but only on the Hilbert-Schmidt distance between them. $D_{\max }$ as a function of $\alpha$ has its largest value for $\alpha=\pi$ i.e. for classical-classical initial state (Fig. 3). For $\alpha=0$ or $2 \pi$, the produced discord is zero, since the corresponding initial states are product states and local time evolution cannot create any quantum correlations in the system [4]. From this plot we see also that all classical-quantum states of this type with non-zero distance between $\rho_{1}^{B}$ and $\rho_{2}^{B}$ (i.e. with non-zero value of $C_{M}$ ) evolve into states with non-trivial quantum correlations. General class of classical-quantum states is much more difficult to study, but the numerical calculations suggests the same behavior of geometric discord.

\section{Conclusions}

We have investigated the possibility of local creation of quantum discord in the system of independent two-level atoms coupled to the vacuum. Individual spontaneous emission of the atoms leads to the local evolution destroying quantum entanglement. At the same time, this kind of evolution can create quantum discord of separable states. We have shown that all classically correlated initial states are driven by the process of spontaneous emission into the states which are still separable but have non-zero quantum discord. The largest value of the created discord is obtained for the classical-classical states (3.10) for which $C_{M}\left(\rho_{c c}\right)=1$ and the corresponding 
orthogonal projectors (3.8) are defined by $\vartheta=\pi / 4$. For the classical-quantum states (3.9) the creation of quantum discord is always less effective.

Open Access This article is distributed under the terms of the Creative Commons Attribution License which permits any use, distribution, and reproduction in any medium, provided the original author(s) and the source are credited.

\section{References}

1. Ollivier, H., Zurek, W.H.: Quantum discord: a measure of the quantumness of correlations. Phys. Rev. Lett. 88, 017901 (2001)

2. Ferraro, A., et al.: Almost all quantum states have nonclassical correlations. Phys. Rev. A 81, 052318 (2010)

3. Streltsov, A., Kampermann, H., Bruss, D.: Behavior of quantum correlations under local noise. Phys. Rev. Lett. 107, 170502 (2011)

4. Hu, X., et al.: Necessary and sufficient condition for Markovian-dissipative-dynamics-induced quantum discord. Phys. Rev. A 84, 022113 (2011)

5. Hu, X., et al.: Necessary and sufficient conditions for local creation of quantum correlation. Phys. Rev. A 85, 032102 (2012)

6. Guo, Y., Hou, J.: Necessary and sufficient conditions for the local creation of quantum discord. J. Phys. A Math. Theor. 46, 155301 (2013)

7. Ciccarello, F., Giovannetti, V.: Creating quantum correlations through local nonunitary memoryless channels. Phys. Rev. A 85, 010102 (2012)

8. Ciccarello, F., Giovannetti, V.: Local-channel-induced rise of quantum correlations in continuousvariable systems. Phys. Rev. A 85, 022108 (2012)

9. Gessner, M., Laine, E.-M., Breuer, H.-P., Piilo, J.: Correlations in quantum states and the local creation of quantum discord. Phys. Rev. A 85, 052122 (2012)

10. Campbell, S., et al.: Propagation of nonclassical correlations across a quantum spin chain. Phys. Rev. A 84, 052316 (2011)

11. Dakić, B., Vedral, V., Brukner, C.: Necessary and sufficient condition for nonzero quantum discord. Phys. Rev. Lett. 105, 190502 (2010)

12. Bellomo, B., et al.: Unified view of correlations using the square-norm distance. Phys. Rev. A 85, 032104 (2012)

13. Derkacz, Ł., Gwóźdź, M., Jakóbczyk, L.: Entanglement beyond tensor product structure: algebraic aspects of quantum non-separability. J. Phys. A Math. Theor. 45, 025302 (2012)

14. Hall, M.J.W.: Correlation distance and bounds for mutual, information, arXiv:1307.2697

15. Piani, M.: Problem with geometric discord. Phys. Rev. A 86, 034101 (2012)

16. Tufarelli, T., et al.: Quantum resources for hybrid communication via qubit-oscillator states. Phys. Rev. A 86, 052326 (2012)

17. Paula, F.M., de Oliveira, T.R., Sarandy, M.S.: Geometric quantum discord through the Schatten 1-norm. Phys. Rev. A 87, 064101 (2013)

18. Ciacarello, F., Tufarelli, T., Giovannetti, V.: Towards computability of trace distance discord, arXiv: 1304.6879

19. Jakóbczyk, L.: Spontaneous emission and quantum discord: comparison of Hilbert-Schmidt and trace distance discord, work in progress

20. Girolami, D., Adesso, G.: Interplay between computable measures of entanglement and other quantum correlations. Phys. Rev. A 84, 052110 (2011)

21. Rana, S., Parashar, P.: Entanglement is not a lower bound for geometric discord. Phys. Rev. A 86, 030302(R) (2012)

22. $\mathrm{Xu}, \mathrm{J} .:$ Geometric measure of quantum discord over two-sided projective measurements. Phys. Lett. A 376, 320 (2012)

23. Jakóbczyk, L.: Maximal mutual correlation: an algebraic measure of correlations in bipartite quantum systems, in preparation

24. Agarwal, G.S.: Quantum Statistical Theories of Spontaneous Emission and their Relation to Other Approaches. Springer, Berlin (1974) 